\title{
Effects of aspect ratios on flow friction and thermal behavior inside a close domain using lattice Boltzmann method
}

\author{
M. A. Taher ${ }^{1}$, Litan Kumar Saha ${ }^{2}$, Y. W. Lee ${ }^{1, *}$ \\ ${ }^{1}$ Dept. of Mechanical and Automotive Engineering, Pukyong National University, Busan 608-739, Korea \\ ${ }^{2}$ Department of Applied Mathematics, University of Dhaka, Dhaka, Bangladesh \\ Email address: \\ taher@pknu.ac.kr (M. A. Taher), lksaha.math@gmail.com (L. K. Saha), ywlee@pknu.ac.kr (Y. W. Lee)
}

\section{To cite this article:}

M. A. Taher, Litan Kumar Saha, Y. W. Lee. Effects of Aspect Ratios on Flow Friction and Thermal Behavior Inside a Close Domain Using Lattice Boltzmann Method. American Journal of Applied Mathematics. Special Issue: Fluid Flow and Heat Transfer Inside a Closed Domain. Vol. 3, No. 1-1, 2015, pp. 1-7. doi: 10.11648/j.ajam.s.2015030101.11

\begin{abstract}
Numerical study of flow friction and thermal behavior on a moving lid of a triangular cavity with various aspect ratios has been discussed using a Thermal Lattice Boltzmann Method (TLBM). To analyze the combined force and free convection, known as mixed convection, flows and heat transfer process in a lid driven triangular cavity with various aspect ratios $\mathrm{AR}(=\mathrm{L} / \mathrm{H})=0.5,1.0$ and 2.0 are taken into account in the present study. The results are presented as stream function in terms of velocity, average heat transfer rate in terms of Nusselt number $(\mathrm{Nu})$, and friction on moving lid of cavity for different Reynolds number (Re) as well as buoyancy parameter $(\lambda)$. The heat transfer rate and friction on moving lid increased and decreased significantly with increasing Reynolds number and aspect ratios but they are linearly and very slowly changed with buoyancy effects. Similarly, the fluid flow behavior significantly changed with higher Reynolds number and aspect ratios compare to lower Re and AR. In addition, the less friction and more heat transfer occurred at case of free convection dominant case compare to forced convection dominant case. Similar behavior is observed for fluid flow analysis inside cavity. The model is validated by other numerical scheme and a very good agreement is found.
\end{abstract}

Keywords: Lattice-Boltzmann, Heat Transfer, Friction Factor, Richardson Number, Reynolds Number, Stream Function

\section{Introduction}

Heat transfer, friction and fluid flows due to influence of free and forced convections, commonly referred to as "mixed convection" flows, are intensively studied since last century.The lid-driven closed cavities are mechanically driven by tangentially moving walls which are represented as a basic problem in convection heat transfer. In most geophysical flows, the temperature difference is the driving mechanism of the motion of the fluid. From a practical point of view, the research on natural, force and mixed convections in a cavity of various shapes with different boundary conditions and numerical techniques are extensively discussed by many researchers [1-5]. Moreover, to the experimental and theoretical investigations, various computational methods are used to study the fluid flow and heat transfer in close domain like cavity flow. The Lattice Boltzmann Method (LBM) is one of the methods available to deal with such kind of problems as a novel alternative to traditional methods. It is based on microscopic models or mesoscopic kinetic equations for fluid. In Computational Fluid Dynamics (CFD), fluid properties, such as density, pressure, velocity and temperature are typically described by the N-S equations, which have nonlinear terms making them too expensive to solve numerically in real time. However, the LBM has demonstrated a significant potential and broad applicability with numerous computational advantages such as the parallel of algorithm, the simplicity in programming, and the ability to incorporate microscopic interactions. Lattice gas models with an appropriate choice of the lattice symmetry in fact represent numerical solutions of the Navier-Stokes equations and therefore are able to describe the hydrodynamics problems discussed by Qian et al. [6]. It is commonly recognized that the LBM can faithfully be used to simulate the incompressible Navier-Stokes (N-S) equations with high accuracy and this lattice BGK (LBGK) model, the local equilibrium distribution has been chosen to recover the N-S macroscopic equations by and Chen et al [7]. However, sometimes it is important to 
simulate thermal effects simultaneously with the fluid flows. Though, the temperature distribution in a flow field is of central interest in heat transfer problems and discuss by many researchers [8-9]. In this model, the temperature can be calculated using an internal energy distribution function whereas the macroscopic density and velocity are still simulated using density distribution function. This thermal Lattice Boltzmann model (TLBM) is successfully implemented with different conditions by many researchers. The more details about TLBM with some examples for different cases have been discussed by authors Mohammad [10] and Succi [11]. The TLBM is applied for simulating mixed convection heat transfer and fluid flow related problems in the recent years. Recently some researchers [12-14] have done their work to simulate the mixed convection flow using this method. Mainly their research interests are seen in a square enclosure with different conditions. However, due to its energy related applications, the research on mixed convection in a triangular cavity with upper moving lid is very important. Some investigations are found using traditional numerical methods, based on N-S equations. To author's knowledge, a few investigations of mixed convection in a triangular cavity with upper moving lid using TLBM are found in the literature. To analyze the important characteristics of flow field and heat transfer on the moving lid of close domain for a wide range of non dimensional parameters namely Reynolds number (Re) and the mixed convection parameter $(\lambda)$ with various aspect ratios (AR) of the domain have been discussed in the present study.

\section{Formulation of the Problems}

\subsection{Lattice Boltzmann Method}

For the flow and temperature fields, the thermal lattice Boltzmann method utilizes two distribution functions, $F_{i}(\vec{x}, t)$ is the particle distribution function (PDF) and $G_{i}(\vec{x}, t)$ is the energy distribution function. For D2Q9 model, the fluid domain is considered in uniform Cartesian cells, call square lattice. Each lattice is connected by nine velocity vectors $\left(\vec{e}_{i}\right)$.Therefore, the discrete form of Boltzmann equation, adding force term with some adjustable coefficients, is called the Lattice Boltzmann equation (LBE) and can be written as:

For flow filed:

$$
\begin{aligned}
& F_{i}\left(\vec{x}+\Delta t \quad \vec{e}_{i}, t+\Delta t\right)-F_{i}(\vec{x}, t) \\
& =-\frac{1}{\tau}\left(F_{i}(\vec{x}, t)-F_{i}^{e q}(\vec{x}, t)\right)+A \vec{e}_{i} \cdot \vec{F}_{a}
\end{aligned}
$$

For temperature filed:

$$
\begin{aligned}
& G_{i}\left(\vec{x}+\Delta t \quad \vec{e}_{i}, t+\Delta t\right)-G_{i}(\vec{x}, t) \\
& =-\frac{1}{\tau_{\theta}}\left(G_{i}(\vec{x}, t)-G_{i}^{e q}(\vec{x}, t)\right)
\end{aligned}
$$

The last term of (1), called the forced term, should introduced be more carefully, where $\mathrm{A}$ is the adjustable coefficient and $\overrightarrow{F_{a}}$ is applied force. $\tau$ and $\tau_{\theta}$ are relaxation time constant for momentum and energy respectively. The relaxation parameter, $\omega=1 / \tau$, depends on the local macroscopic variables, $\rho$ and $\rho \vec{u}$. These variables should satisfy the following laws of conservation:

$$
\rho=\sum_{i} F_{i} \text { and } \rho \vec{u}=\sum_{i} \vec{e}_{i} F_{i}
$$

For two dimensional D2Q9 model, the equilibrium distribution functions for momentum $\left(F_{i}^{e q}\right)$ and energy ( $G_{i}^{e q}$ ) with lattice position $\vec{x}$ and time $t$ are defined by Qian et al. [6], He et al. [8] as:

The governing equations (1)-(4) are non-dimensionalized using the following dimensionless variables:

$$
F_{i}^{e q}=\rho w_{i}\left[1+\frac{3}{c^{2}} \vec{e}_{i} \cdot \vec{u}^{e q}+\frac{9}{2 c^{4}}\left(\vec{e}_{i} \cdot \vec{u}^{e q}\right)^{2}-\frac{3}{2 c^{2}} u^{e q^{2}}\right]
$$

And

$$
G_{i}^{e q}=\varepsilon w_{i}\left[1+\frac{3}{c^{2}} \vec{e}_{i} \cdot \vec{u}^{e q}+\frac{9}{2 c^{4}}\left(\vec{e}_{i} \cdot \vec{u}^{e q}\right)^{2}-\frac{3}{2 c^{2}} u^{e q^{2}}\right]
$$

For D2Q9 model, $w_{0}=4 / 9, w_{\mathrm{i}}=1 / 9, \mathrm{i}=1,2,3,4$, and $w_{i}=$ $1 / 36, i=5,6,7,8$. In presence of any interaction or any external forces, the velocity should be modified by the force term when calculating the equilibrium distribution functions as

$$
\vec{u}^{e q}=\vec{u}+\frac{\tau \vec{F}_{a}}{\rho}
$$

The form of this equilibrium distribution function must be chosen so that the fluid mass and momentum are conserved. The internal energy variable $(\varepsilon)$ of the fluid components is defined as:

$$
\mathcal{E}(x, t)=\sum_{i} G_{i}(x, t)
$$

It is noted that the internal energy, $\mathcal{E}$, is related (proportionally) to the temperature by the thermodynamic relation $\varepsilon=\rho c_{p} T$. Therefore, the mean temperature of the fluid in this model can be written as

$$
T=\frac{\varepsilon(x, t)}{\rho c_{p}}=\frac{\sum_{i} G_{i}(x, t)}{\rho c_{p}}
$$

It is known that the above equations recover the N-S equations both for velocity and temperature fields as reported He et al. [8], if the viscosity coefficient $\mu$ and thermal conductivity $\kappa$ can be identified as 


$$
\mu=\left(\tau-\frac{1}{2}\right) \rho R T \text { and } \kappa=\left(\tau_{\theta}-\frac{1}{2}\right) \rho c_{p} R T
$$

These can be written in lattice unit as $v=\left(\tau-\frac{1}{2}\right) C s^{2}$ and $\alpha=\left(\tau_{\theta}-\frac{1}{2}\right) C s^{2}$ respectively. Here $T$ is the non dimensional temperature. Note that the density distribution functions, the relaxation time for both momentum and energy equations have already been dimensionless quantities. Here, $x$ and $y$ are normalized by $N x$ and $N y$ respectively, where $N x$ is the lattice node in $x$-direction and $N y$ is the lattice node in $y$-direction. The velocity $u$ and $v$ by the characteristic speed $U$. Throughout the simulation, all units are considered as lattice units. The computations were carried out with a code developed by the authors and written in FORTRAN language. The microscopic quantities for momentum $\left(F_{i}\right)$ and energy $\left(G_{i}\right)$ can be evaluated by solving the equations (1) and (2) with other LBM approaches. And therefore, the macroscopic quantities can be calculated in terms of microscopic quantities as shown in equations (3) and (8).

\subsection{Problem Description}

Consider a two dimensional triangular lid driven cavity flow with different aspects ratios. The system consists of a triangular cavity of height $\mathrm{H}$ and length $\mathrm{L}$, whose upper horizontal wall moves from left to right with a constant velocity, while the other inclined walls are fixed. The moving lid is considered to be heated $\mathrm{T}_{\mathrm{H}}$ whereas the inclined walls are assumed to be cold $\mathrm{T}_{\mathrm{C}}$. The cavity aspect ratios are considered at $\mathrm{AR}(=\mathrm{L} / \mathrm{H})=0.5,1.0$ and 2.0 as shown in Figs. 1(a)-(c) with corresponding uniform grids $101 \times 51,101 \times 101$ and $101 \times 201$ respectively.

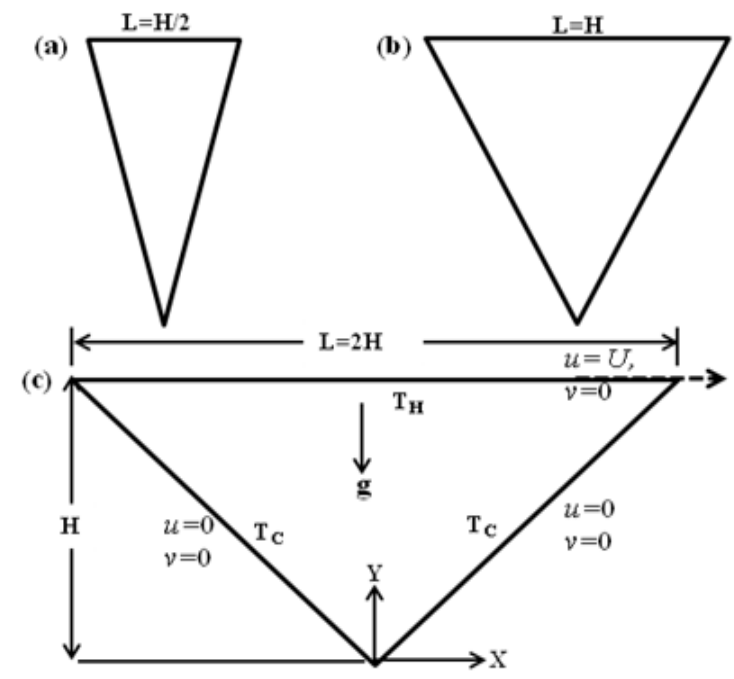

Fig. 1. Physical configuration of the problem with boundary conditions for various aspect ratios $A R(=L / H):$ (a) $A R=0.5$, (b) $A R=1.0$ and (c) $A R=2.0$, same boundary conditions for all cases.
The working fluid is considered as air whose Prandtl number, $\operatorname{Pr}=0.713$ and viscosity, $v=15.11 \times 10^{-6} \mathrm{~m}^{2} / \mathrm{s}$. For convection, the momentum and energy equations are coupled and the flow is driven by temperature or mass gradient, i.e. buoyancy force. Hence there is an extra force term that needs to be considered in solving LB equations. Under Boussinesq approximation, the force term per unit mass can be written as $\vec{F}_{a}(\vec{x}, t)=\rho(\vec{x}, t) g \beta\left(T(\vec{x}, t)-T_{r e f}\right)$, where $\mathrm{T}_{\text {ref }}$ is the reference temperature of the fluid, $g$ is the gravitation acceleration, $\beta$ is the thermal expansion coefficient. For small temperature difference, the buoyancy force is balanced by viscous drag and heat dissipation. The ratio of the buoyancy force to the product of viscous force and heat diffusion rates defines the Rayleigh number, $\operatorname{Ra}=\operatorname{Pr} \times \mathrm{Gr}=\frac{\mathrm{g} \beta \Delta T H^{3}}{v \alpha}$, where, $\mathrm{Gr}=\frac{\mathrm{g} \beta \Delta T H^{3}}{v^{2}}$ is the Grashof number, $\operatorname{Pr}=\mathrm{v} / \alpha$ is the Prandtl number. In fluid mechanics, the Grashof number divided by the square of the Reynolds number is called buoyancy parameter, i.e. $\lambda=\frac{G r}{\operatorname{Re}^{2}}$, sometimes it is called mixed convection parameter or Richardson number (Ri). The Reynolds number, $\operatorname{Re}=\frac{\rho H U}{\mu}$, is another important parameter to characterized the flow friction, where $U$ is the upper moving lid velocity and $H$ is the characteristic height of the cavity. Therefore, the force is induced by the moving lid, which shears the surface layer of the fluid inside the cavity by creating a recalculating flow. The effect of buoyancy becomes insignificant i.e. the force convection is dominant to free convection when $0.1 \leq \lambda<1$ and if $\lambda<0.1$, the free convection may be neglected. On the other hand, the buoyancy effect becomes more significant, i.e. free convection is dominant to forced convection if $1<\lambda \leq 10$ and the force convection is neglected for $\lambda>10$. More typically both forced and natural convections need to be taken into account when for $\lambda=1$.

\section{Results and Discussions}

The macroscopic variables of interest (i.e. density, velocity, temperature, pressure etc.) can easily be obtained from microscopic particle distribution function for momentum $\left(F_{i}\right)$ and energy $\left(G_{i}\right)$ by solving the equations (1) and (2) with other LBM approaches. The main characteristics of the fluid flow and energy transport for the case of uniformly heated top moving lid with symmetrical cold inclined walls are shown in the following figures.

\subsection{Code Validation}

The validation of present simulation has been shown against the numerical results of Chen et al. [4]. The variation of average heat transfer rate in terms of Nusselt number, $\mathrm{Nu}$, for different values of Reynolds number on the moving lid of the triangular enclosure with $\mathrm{AR}=2.0$ is illustrated in Fig. 2 


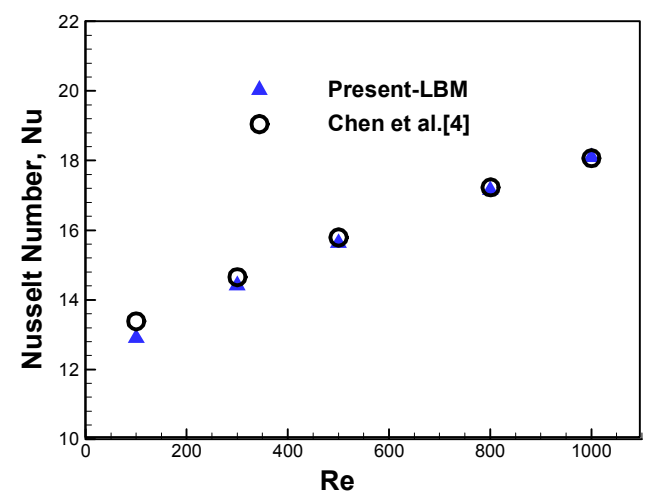

Fig. 2. Average Nusselt number (Nu) as a function of Reynolds number for $\mathrm{Pr}$ $=0.71, A R=2.0$.

It can be seen from the above figure that our present LBM simulations are in very good agreement with numerical solutions of finite-volume method obtained by Chen at al. [4]. It is noted that, for verification of our method the top moving lid is maintained at cold temperature while the lower two inclined walls are uniformly heated. However, it is considered in the present study that the system consists of a cavity heated form above by maintain the lid of the cavity at a constant temperature $\mathrm{T}_{\mathrm{H}}$ higher than the two inclined walls temperature $\mathrm{T}_{\mathrm{C}}$.

\subsection{Flow Friction Analysis}

The fluid resistance is one of the research interests in fluid mechanics in order to investigate the fluid flow behavior in a lid driven cavity. The local friction factor on the moving lid is defined in terms of the local shear stress as [4]:

$$
S_{F x}=\frac{\tau_{x}}{\rho U^{2}}=\left.\frac{\mu}{\rho U^{2}} \frac{\partial u}{\partial y}\right|_{\text {moving lid }}
$$

Based on the local friction, the average friction on the moving lid surface can be further calculated by integration as:

$$
A S_{F}=\frac{1}{L} \int_{0}^{L} S_{F x} d x
$$

Where, $\mathrm{L}$ is the total number of lattice in $\mathrm{x}$-direction. The friction factor on the moving lid of the triangular enclosures is illustrated in figures 3-4 for different non dimensional parameters.

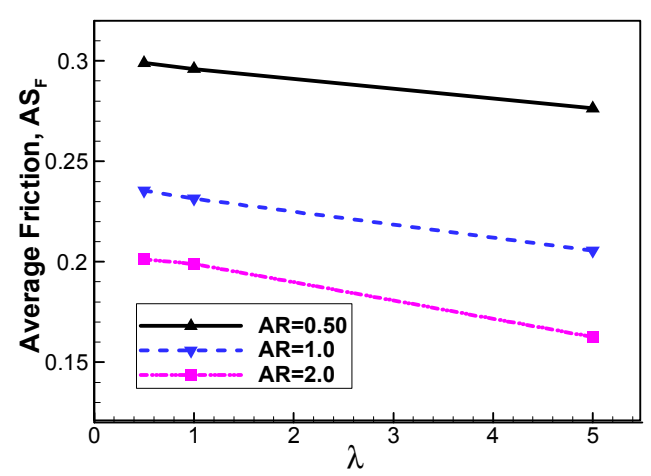

Fig. 3. Buoyancy effect on the average friction on the moving lid for different aspect ratios, $A R=0.5,1.0$ and 2.0, with $R e=300$.

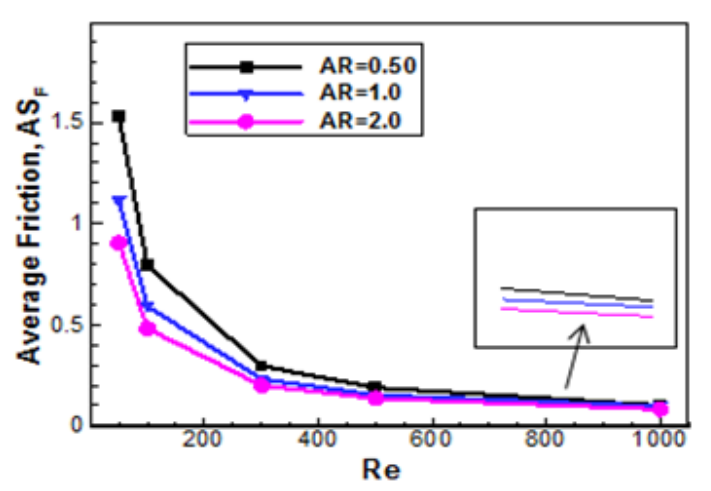

Fig. 4. Average friction as a function of Reynolds number for aspect ratios $A R=0.5,1.0$ and 2.0 with $\lambda=1.0$.

From the above figure is it seen that the average friction on the moving lid decreased linearly and slowly with buoyancy effect but it is significantly decreased with increasing aspect ratios as well as Reynolds number. Specially, in Fig.4, for low Reynolds number, the friction occurred more compare to high Reynolds number. This is obvious because the lid velocity depends on Reynolds number.

\subsection{Heat Transfer Performance}

A usual means of characterizing heat transfer is to calculate the Nusselt number. Actually, the Nusselt number, $\mathrm{Nu}$, is a dimensionless form of the heat transfer coefficient. To investigate the heat transfer performance in terms of effective thermal conductivity of the fluid, the local Nusselt number, $\mathrm{Nu}_{\mathrm{x}}$, is defined by Chen et al. [4].

$$
N u_{x}=\frac{h_{x} L}{k}=\left.\frac{L}{T_{H}-T_{C}} \frac{\partial u}{\partial y}\right|_{\text {moving lid }}
$$

Here $\mathrm{T}$ stands for non-dimensional temperature and $\mathrm{h}_{\mathrm{x}}$ is the local heat transfer coefficient. Therefore, the average Nusselt number on the moving lid is calculated by integration as:

$$
N u=\frac{1}{L} \int_{0}^{L} N u_{x} d x
$$

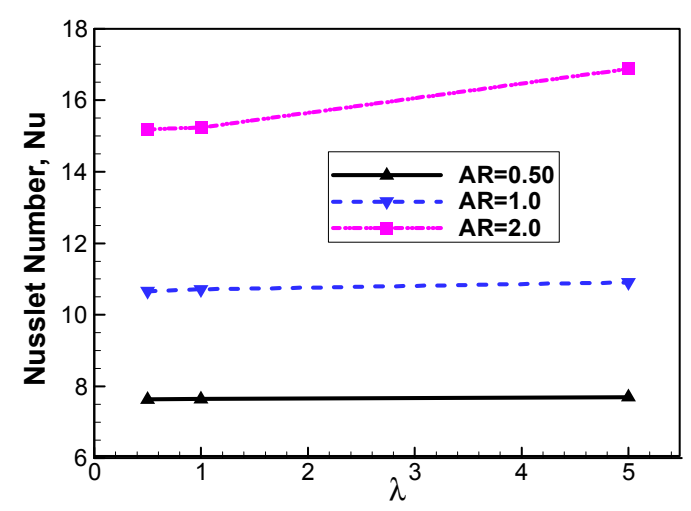

Fig. 5. Variation of the average heat transfer rate in terms of Nusselt number (Nu) with buoyancy parameter ( $\lambda$ ) for different $A R=0.5,1.0$ and 2.0 with $R e=300$. 


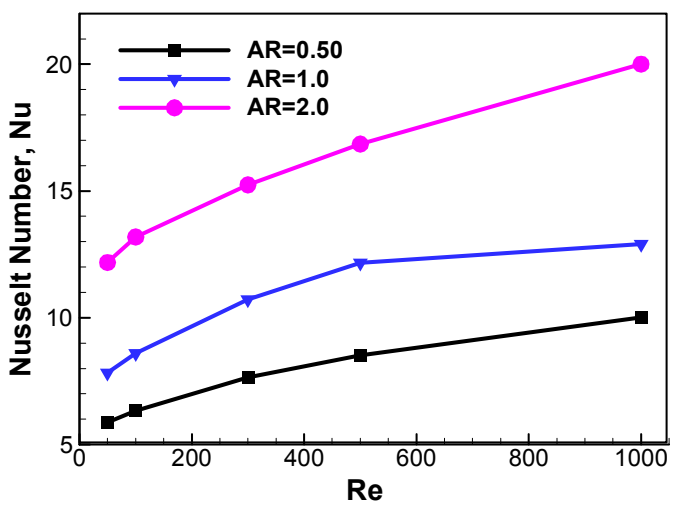

Fig. 6. Variation of the average heat transfer rate in terms of Nusselt number (Nu) with mixed convection parameter (Ri) for different $A R=0.5,1.0$ and 2.0 with $\lambda=1.0$.

The average rate of heat transfer in terms of Nusselt number $\mathrm{Nu}$ ) on the moving lid of the triangular cavity for different Reynolds number and aspect ratios with buoyancy effects are explained in figures 5-6. It is seen from Fig.5 that the heat transfer rate increased very slowly with buoyancy parameter at constant Reynolds number, especially for lower values of $\lambda$, forced convection dominant case compare to free convection dominant case, $\lambda>1$. This phenomenon is clearly seen for high aspect ratio, Fig.5, square symbols line. With increasing the aspect ratios, the heat transfer performances significantly increased. Moreover, it is observed from Fig. 6 that the magnitude of the local Nusselt number is increased significantly with increasing the Reynolds number.

\subsection{Flow and Thermal Filed Analysis}

In order to investigate the deeper insight into the effects of aspect ratios of lid driven close domain, buoyancy parameter $(\lambda)$, or mixed convection parameter with Reynolds number $(\mathrm{Re})$, the velocity profiles in terms of streams lines with velocity vectors inside the cavity are presented in Figs.7-9.
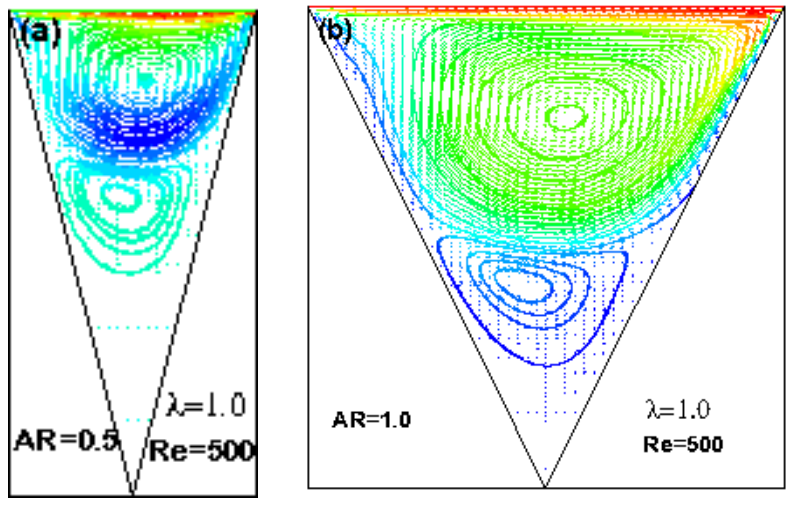

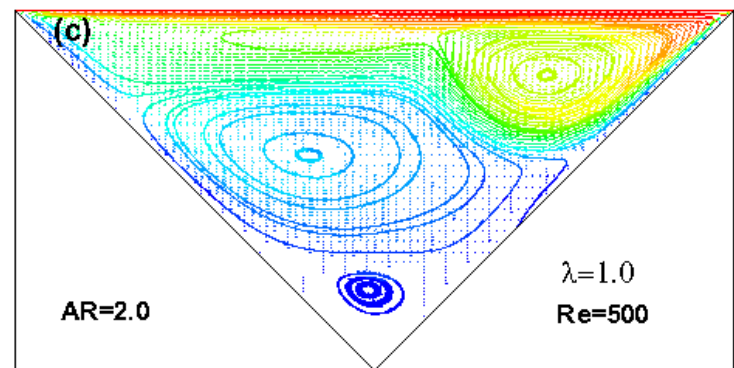

Fig. 7. Streamline contours with velocity vectors for various $A R$ at $R e=500$ and $\lambda=1.0$.

Fig. 7 indicated the aspect ratio effects on flow field in terms of steam lines for constant Reynolds number, $\mathrm{Re}=500$ and $\lambda$ $=1.0$. A primary and a secondary recirculation zones are observed for $\mathrm{AR}=0.5$ and 1.0. It is clearly seen that the size and shape of recirculation zones are dependent on the cavity size. The size and shape of secondary cell also increased with increasing the aspect ratio and finally some more vortices are seen inside the cavity. Similar phenomenon is seen in Fig.8. The stream contours for different cases of buoyancy parameter, $\lambda=0.5,1.0$ and 5.0 at fixed $\mathrm{Re}=500$ and $\mathrm{AR}=1.0$ are seen in Fig.8. For low values of $\lambda$, the forced convection dominant case, a primary and a secondary recirculation zones are observed. The large circulating cell spreads over the space inside the cavity by strengthening its circulation. However, in the case of free convection dominant, a tertiary cell is seen at the bottom of close domain. Fig.9. at very low Reynolds number, one circulating cell is occurred with geometrical size and shape 9(a). Further increase in the Reynolds number, Fig. 9(b)-(c), another secondary cell at the bottom of the cavity is observed. The size and shape of secondary cell increases with increase in the Reynolds number and thereafter the flow starts to lose the symmetrical structure and finally multiple cells are seen inside the cavity with different size and shape. Moreover, the size and shape are not of regular form and also the vortex centre moves with Reynolds number due to the effect of moving lid.
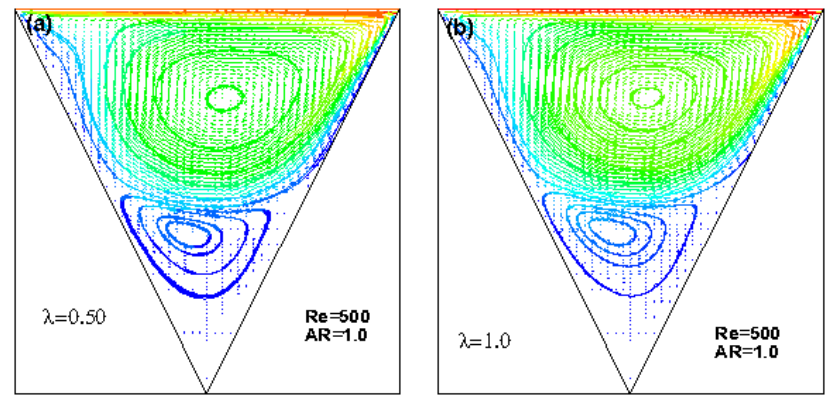


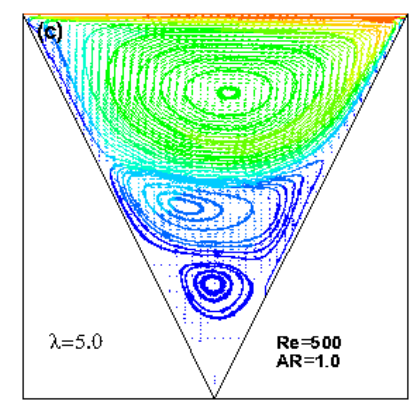

Fig. 8. Streamline contours with velocity vectors for various $\lambda$ at $R e=500$ and $A R=1.0$.
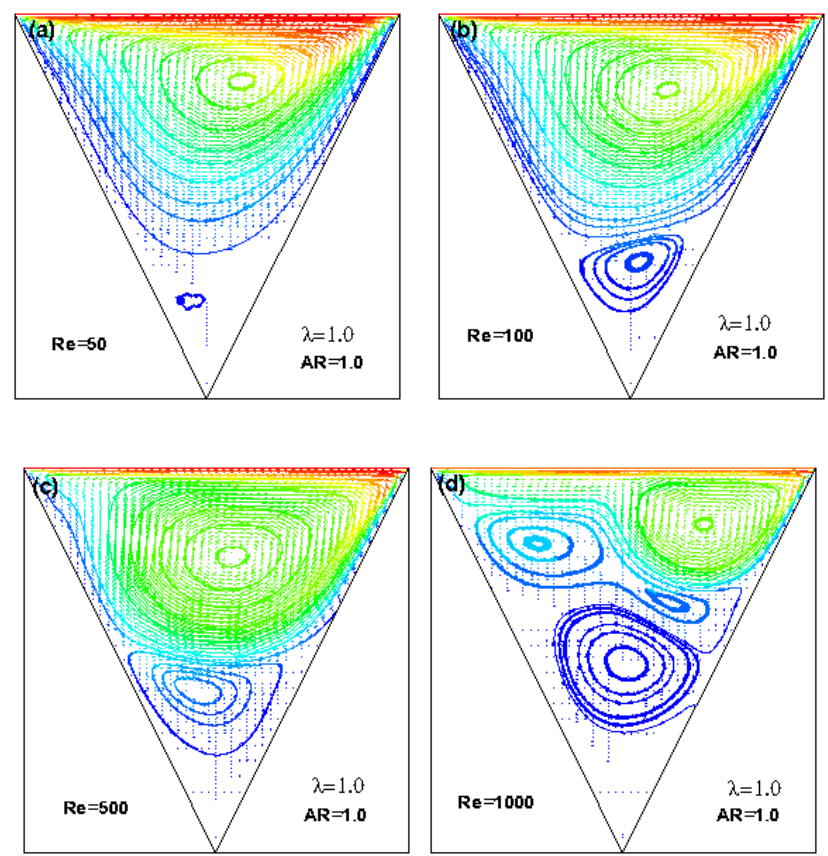

Fig. 9. Streamline contours with velocity vectors for various $R e$ at $\lambda=1.0$ and $A R=1.0$.

The corresponding temperature distributions in terms of isotherms are described in the following Figs.10-12. Fig.10 indicated the effects of aspect ratios on isotherms for $\mathrm{Re}=500$ and $A R=1.0$. It is seen that the hot fluid mixing zone increased with aspect ratio. Fig.10(c), as the vortex motion produces a convection effect in the area near the right corner, the thermal stratification is obviously seen inside the cavity. In Fig.11, the temperature contours for different values of mixed convection parameter, $\lambda=0.5$ to 5.0 with $\mathrm{AR}=1.0$ and $\mathrm{Re}=500$ are discussed. In this case, with increasing the values of $\lambda$, natural convection is dominant, therefore, the buoyancy force significantly enhanced on the isotherms inside the domain. As a result, the fluid temperature increases significantly and it has shown a tendency to concentrate at upper half of cold inclined walls inside the cavity. The Reynolds number effect on isotherms is illustrated in Fig.12. Due to increasing the Reynolds number, the moving lid also increased. Therefore, the cold fluid mixes well with the arisen cold fluid from bottom and higher heat transfer rate is observed near at the upper right corner of the cavity. These phenomena of mixed convection in a lid driven cavity flow are intensively used in various fields of science and engineering.
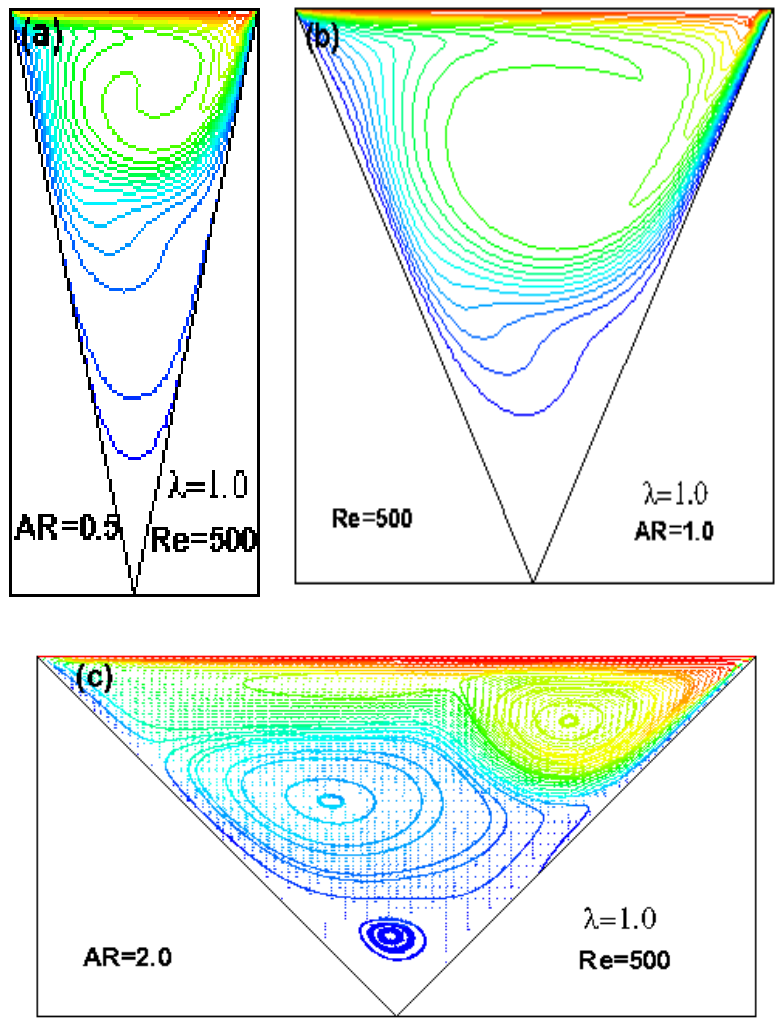

Fig. 10. Isotherms for various $A R$ at $R e=500$ and $\lambda=1.0$.
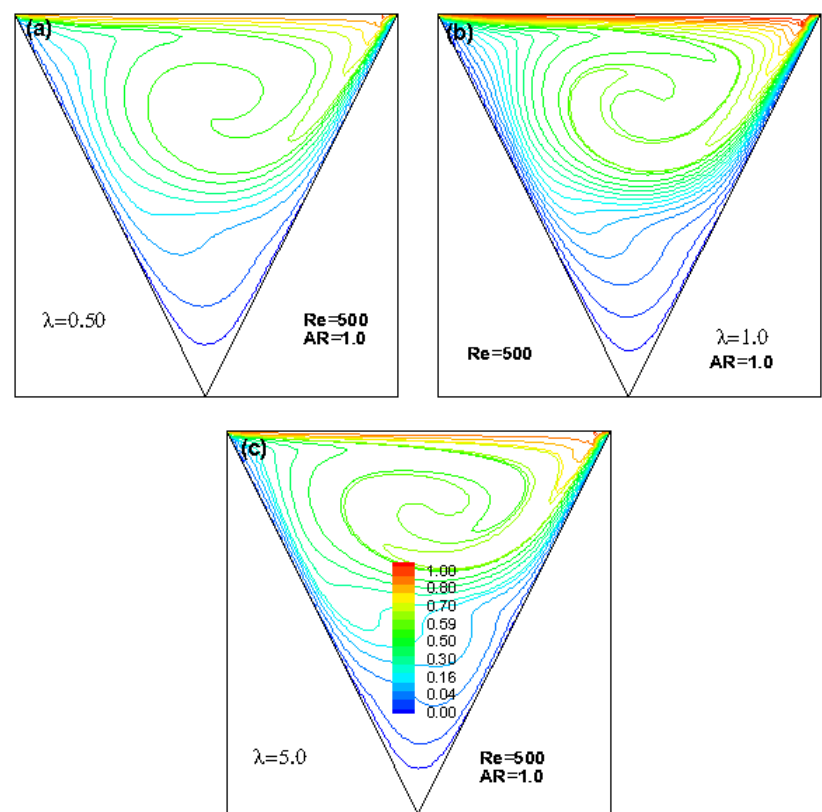

Fig. 11. Isotherms for various $\lambda$ at $R e=500$ and $A R=1.0$. 

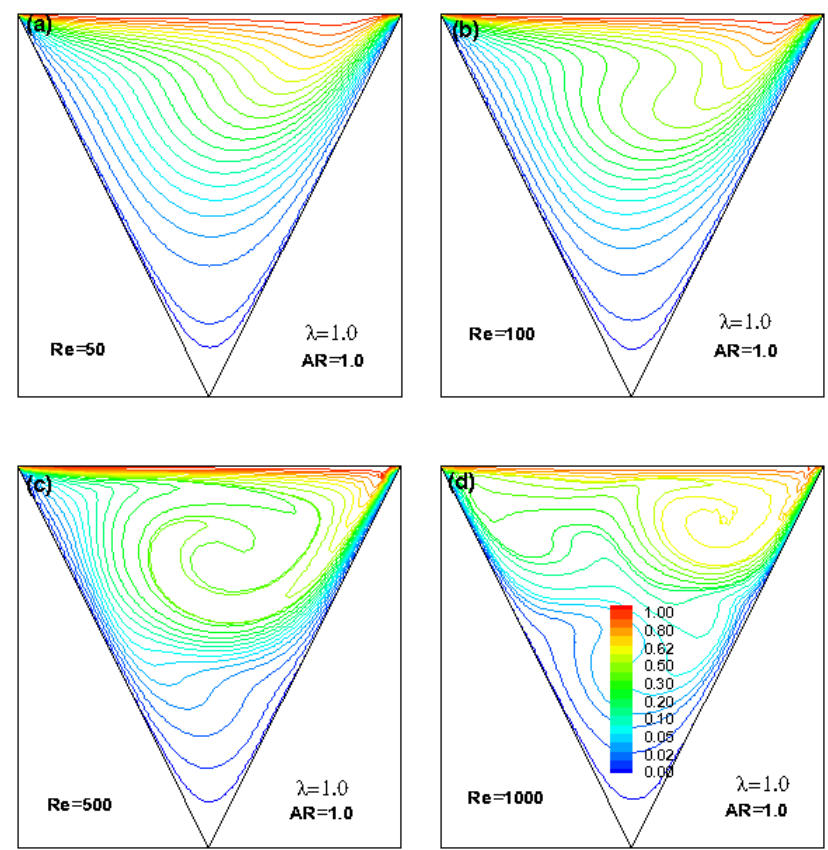

Fig. 12. Isotherms for various Re at $\lambda=1.0$ and $A R=1.0$.

\section{Conclusion}

To analyze the friction factor and heat transfer characteristics in a lid driven triangular enclosure with different aspects ratios and dimensionless parameter are studied using TLBM. It is seen that the mixed convection cavity flow problems are suitably applied in this method. The following results are noted during the investigation:

- The average friction is decreased linearly with increasing buoyancy effect but it is significantly decreased with increasing the aspect ratios as well as Reynolds number due to moving lid effect. The maximum friction occurred for very low Reynolds number and low aspect ratios.

- The rate of heat transfer increased significantly with increasing Reynolds number and aspect ratios.

- However, with increasing the buoyancy parameter the heat transfer rate increased very slowly compare to that of Reynolds number and aspect ratios.

- In addition, for free convection dominant case, $\lambda>1$, the less friction and more heat transfer rate are seen compare to forced convection dominant case, $\lambda \leq 1$.

- A central vortex is seen for low Reynolds numbers. However, multi-cellular flow becomes evident for higher Reynolds number and aspect ratios as well as free convection dominant case. And consequently the isotherms significantly enhanced the fluid inside the cavity.

- In addition, for higher Reynolds number, the higher heat transfer area is located near the right corner of the cavity. This is because of the centre of vortex moves toward the right corner due to moving lid. Similar phenomenon is observed at the case of high aspect ratio.

\section{References}

[1] C. Sun, B. Yu, H. F. Oztop, Yi. Wnag, and J. Wei, "Control of mixed convection in lid-driven enclosures using conductive triangular fins", Int. J. Heat and Mass Transfer, Vol. 54, pp. 894-909, 2011.

[2] M. Li, and T. Tang, "Steady viscous flow in a triangular cavity by efficient numerical techniques", Computers Math. App.,Vol. 31, No.10, pp.55-65, 1996.

[3] M. Ahmed, and H. C. Kuhlmann, "Flow instability in triangular lid-driven cavities with wall motion away from a rectangular corner", Fluid Dyn. Res., Vol.44, pp.025501-21, 2012.

[4] C-L Chen, and C-H.Cheng, "Numerical study of flow and thermal behavior of lid-driven flows in cavities of small aspect ratios", Int. J. Num. Methods in Fluid, Vol.52, pp.785-799, 2006.

[5] T. Basak, G. Aravind, and S. Roy, "Visualization of heat flow due to natural convection within triangular cavities using Bejan's heatline concept", Int. J. of Heat and Mass Transfer, Vol.52, pp.2824-2833, 2009.

[6] Y. H. Qian, D. D'Humieres, and, P. Lallemand, "Lattice BGK models for Navier- Stokes equation", Euro physics letter, Vol. 17, No.6, pp. 479-484, 1992.

[7] H. Chen, S. Chen, and W. H. Matthaeus, "Recovery of the Navier-Stokes equations using a lattice-gas Boltzmann method", Physical Review A, Vol. 45, No.8, pp. 5339-5342, 1992.

[8] X. He, S. Chen, and G. D. Doolen, "A novel thermal model for the Lattice Boltzmann method in incompressible limit", J. Comp. Physics, Vol.146, pp. 282-300, 1988.

[9] M. A. Taher, S. C. Saha, Y. W Lee, and H. D. Kim, "Numerical study of lid driven square cavity with heat generation using LBM", Americal Journal of Fluid Mechanics, Vol.3, No.2, pp.340-344, 2013.

[10] A. Mohammad, Applied lattice Boltzmann method for Transport phenomena, momentum, heat and mass transfer, The University of Calgary, Albetra, Canada, 2007.

[11] Succi, S., The lattice Boltzmann equation for fluid dynamics and beyond, Oxford University, UK, 2001.

[12] A. R. Darzi, M. Farhadi, K. Sedighi, E. Fattahi, and H. Nemati, "Mixed convection simulation on inclined lid driven cavity using Lattice Boltzmann method", IJST, Transactions of Mechanical Engineering, Vol. 35, No. M1, pp.73-83, 2011.

[13] M. Jafari, M. Farhadi, K. Sedighi, and E. Fattahi, "Numerical simulation of convection heat transfer in a lid driven cavity with an open side", World Academy of Science, Engineering and Tecnology, Vol.5, No.11, pp.291-295, 2011.

[14] Z. Guo, and T. S. Zhao, "A Lattice Boltzamnn model for convection heat transfer in porous media", Numerical Heat Transfer, Part B, Vol.47, pp.157-177, 2005. 